\title{
Simulation of a Broadcasting Method Based on Busy Tone
}

\author{
Cao Wen-jing, Han Qing-tian, Xu Sheng-hong
}

Department of Control Engineering , Naval Aeronautical and Astronautical University , Yantai 264001 , China

caowenjing751215@sohu.com

\author{
Keywords: Ad hoc network, Broadcasting method, Busy tone, Simulation
}

\begin{abstract}
When the receiving nodes are uncertain or the topology of the ad hoc network is high dynamic, broadcasting is an effective data dissemination method. In this research, a broadcasting method based on busy tone is modeled and implemented with the network simulation platform OPNET, and the performance of the broadcasting method is analyzed based on experimental results. Experimental results show that, in the network with various load, performance of the broadcasting method based on busy tone is good.
\end{abstract}

\section{Introduction}

When the receiving nodes are uncertain or the topology of the ad hoc network is high dynamic, broadcasting is an efficient technique to exchange information. In this paper, the broadcasting method based on busy tone is modeled and implemented with OPNET network simulation platform, and its performance is analyzed.

\section{A broadcasting method based on busy tone}

Currently, the most widely used channel reservation mechanism is RTS/CTS handshake. For example, in BSMA protocol, BWM protocol, BMMM protocol, LAMM protocol etc ${ }^{[1-7]}$, RTS/CTS handshake is used to reserve channel. The cost of RTS/CTS handshake is increased network load of transmitting a lot of control packets.

The assumption of RTS/CTS mechanism is that all the related nodes can receive RTS/CTS control package. But this assumption is not always true for the mobile ad hoc network in which the relative position of nodes changes rapidly. RTS/CTS handshake has following disadvantages:

(1) Due to the rapid changing relative position of nodes, a complete handshake between source node and destination node is difficult to completed, which lead to unexpected access channel delay.

(2) RTS/CTS handshake not only notifies the nodes in one-hop range of source node with RTS, but also notifies the nodes in two hops range of source node with CTS the coming broadcasting. Therefore, RTS/CTS handshake reserves two hops range channel of source node. In the high density network, reserve two hops would lead to a large number of nodes waiting to access the common channel.

In a word, reserve channel with RTS/CTS handshake is complex and the access channel delay is long in high density network.

To overcome the problems caused by RTS/CTS handshake, referring to some unicast methods, this research designed a single-hop broadcasting method based on busy tone. In this method, busy tone is used to reserve the one hop channel of source node, the broadcasted data message is used to indicate the one hop channel state of source node.

In the single-hop broadcasting method based on busy tone, transmitter and receiver both have two channels: the channel transmits data message is called data channel, and the other channel transmits busy tone is called busy tone channel.

Following is the operation process of the single-hop broadcasting method:

(1) Node detects data channel and busy tone channel through receiver:

1) If data channel is busy, then receive data message. Receive data message over, continue to detect data channel and busy channel. 
2) If busy tone channel is busy and data channel is free, which means that there is other node in one hop range is reserving channel, continue to detect data and busy tone channel.

3) If data channel is free, busy tone channel is free, and the node itself has data to broadcast, then start to reserve channel.

4) If data channel is free, busy tone channel is free, and the node itself has no data to broadcast, then continue to detect data and busy tone channel.

(2) The process of reserve channel is:

Set Timer $\mathrm{t}, \mathrm{t}=\mathrm{d} / \mathrm{c}$, where $\mathrm{d}$ is the one hop range of wireless signal, $\mathrm{c}$ is the propagation velocity of wireless signal, and $t$ is the time span of wireless signal propagate one hop range. Broadcast busy tone, detect data and busy tone channels:

1) If data channel is busy before time $t$ out, then stop broadcasting busy tone, and start to receive data message.

2) If data channel is free, and there's busy tone from other node before time t out, then cancel Timer, stop broadcasting busy tone, back off for a time span t', and continue to detect channel.

3) If data and busy tone channels are free before time $t$ out, then the node reserves channel successfully. The node starts to broadcast data. When broadcasting data message is over, continue to detect data and busy tone channel.

\section{Modeling and implementation of the broadcasting method based on busy tone with OPNET platform}

OPNET is one of the most widely used network simulation platform. Modeling with OPNET consists three parts: network modeling, node modeling and process modeling. Network model describes network topology, geographical layout, and mobility, etc. Node model describes various kinds of calculation and communication devices in the network. Process model describes the communication operation of node, such as protocol, algorithm, etc. In a word, the main task of network simulation is to design network model, node model, and process model. The design and implement of broadcasting method is to design corresponding node model and process model.

Node model design. Node model is to describe node's function. A node is usually made up of many modules and each module plays one part of the node function, such as data generation and storage, etc. To simulate the broadcasting method, in this research, the node model comprises six modules as shown in figure 1.

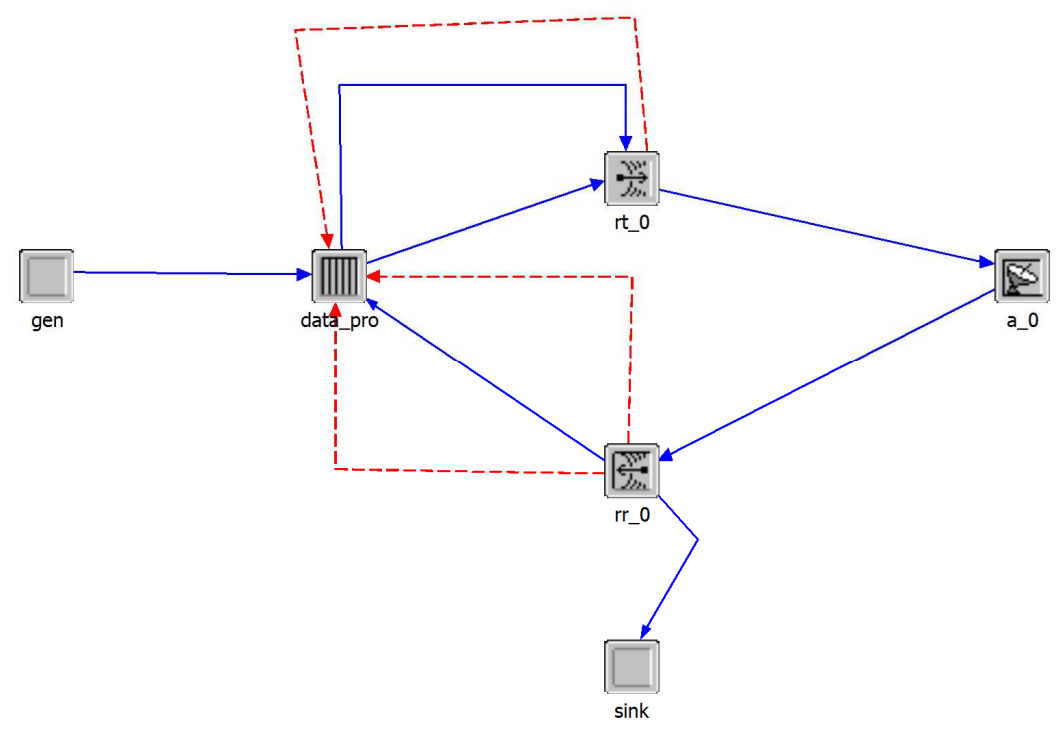

Figure 1. Node model

(1) Module gen is the module to generate data packet.

(2) Module rt_ 0 is a wireless transmitter, and rr_0 is a wireless receiver.

The wireless transmitter and receiver have two channels: data channel and busy tone channel. 
(3) Module a 0 is an antenna.

(4) Module data pro is the module to implement the broadcasting method. Following is the functions of the module:

1) Receive the data from module gen, and choose proper time to transmit it to the data channel of $\mathrm{rt} 0$.

2) Reserve channel with busy tone.

3) Receive data from rr_0.

4) Detect data channel and busy tone channel by rr_0.

5) Detect data channel by rt_0.

(5) Sink is the module to destroy the busy tone packet received from rr_0.

Simulation of busy tone. In OPNET, packet transmission is used to simulate information flow in practical network. Theoretically, busy tone is analog signal looking like sine wave. To design simulation model of the broadcasting method based on busy tone with OPNET platform, it is critical to simulate busy tone with packet transmission. To stop busy tone transmission and free channel on time, small packet is used to simulate busy tone. Here, suppose busy tone channel data rate of transmitter and receiver is set as $10^{6} \mathrm{bps}$, busy tone packet length is 1 bit. To simulate continuous busy tone signal, the interval time to generate and send busy tone packet is $10^{-6}$ seconds.

Process design. Each module in node model is implemented by a process model. Following we design data_pro module, which implements the broadcasting method based on busy tone. With OPNET, algorithm is described as state transition machine. To develop state transition machine, following steps are carried out:

(1) Abstract the states of the protocol process, making sure that these states are mutually exclusive and complementary. The process can only be at one state at one time.

(2) Determine the possible event in the process, construct events response table, and final establish state transition machine.

Abstract states and events of the process. State is a module of a process. States are mutually exclusive and complementary. Process should be at only one state at one time, and all states make up of the process state space. During simulating, the process keeps transiting from one state to another. Based on the operation principle, process of the broadcasting method based on busy tone includes six states, which are listed in table 1.

Table 1. States of the process

\begin{tabular}{|l|l|}
\hline State & State Description \\
\hline INIT & Process initiate \\
\hline IDLE & Waiting for event \\
\hline CONTEND & Node reserve channel \\
\hline SENDDATA & Broadcast data packet \\
\hline RECEPT & Receive data packet \\
\hline BACKOFF & Back off for a time span \\
\hline
\end{tabular}

Event is the motivation to state transition. Each event is an interrupt and has an interruption type. According the operation process of the method, events of the process are listed in table 2.

Table 2. Events and corresponding interrupts of the process

\begin{tabular}{|l|l|l|}
\hline Name of Event & Event Description & Interrupt Type \\
\hline PK_ARRIVAL_FROMGEN & Data generated by gen module arrives & Stream interrupt \\
\hline PK_ARRIVAL_FROMRR & Data received by rr_0 module arrives & Stream interrupt \\
\hline DATACHANNEL_BUSY & $\begin{array}{l}\text { State of data channel detected by rr_0 changes from free to } \\
\text { busy }\end{array}$ & $\begin{array}{l}\text { Statistical } \\
\text { interrupt }\end{array}$ \\
\hline DATACHANNEL_FREED & $\begin{array}{l}\text { State of data channel detected by rr_0 changes from busy to } \\
\text { free }\end{array}$ & $\begin{array}{l}\text { Statistical } \\
\text { interrupt }\end{array}$ \\
\hline TDATACHANNEL_FREED & $\begin{array}{l}\text { State of data channel detected by rt_0 changes from busy to } \\
\text { free }\end{array}$ & $\begin{array}{l}\text { Statistical } \\
\text { interrupt }\end{array}$ \\
\hline BACKOFF_OVER & Reserve channel time is out & Self interrupt \\
\hline BACKOFF1_OVER & Back off time is out & Self interrupt \\
\hline INTER_BT_SELFINTRPT & The timer of sending busy tone expires & Self interrupt \\
\hline
\end{tabular}


Establish state transition machine. According to the broadcasting method operation process, process states, process interrupts, state transition machine could be established, which is shown in figure 2. In figure 2, red state is unforced and green state is forced. Unforced state can stop the process running, while forced state can not stop the process running. Interrupts motivate the process transit from one unforced state to another unforced state. The initial unforced state is IDLE.

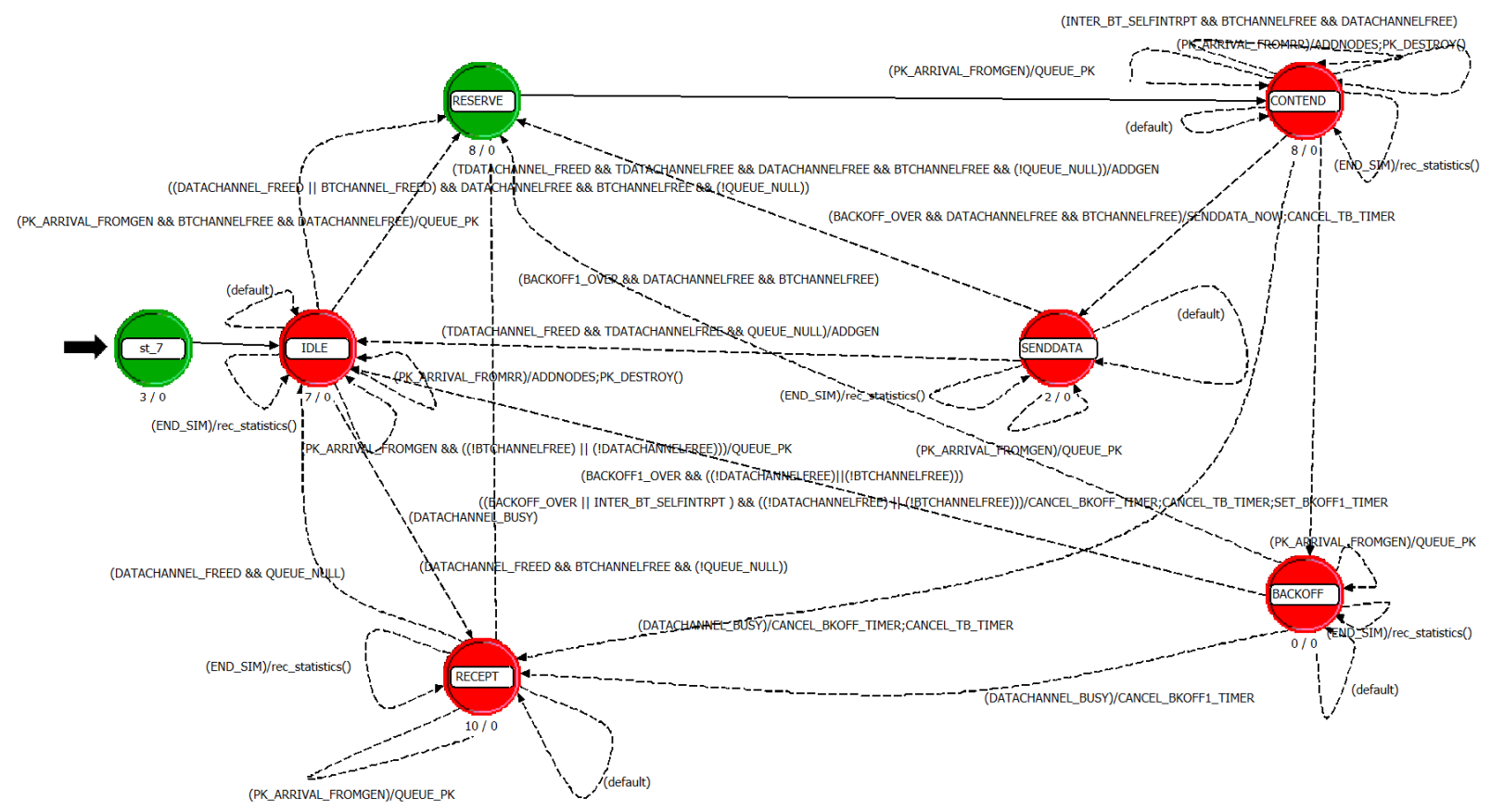

Figure 2. State transition machine of the process model

\section{Experiment design and performance analysis}

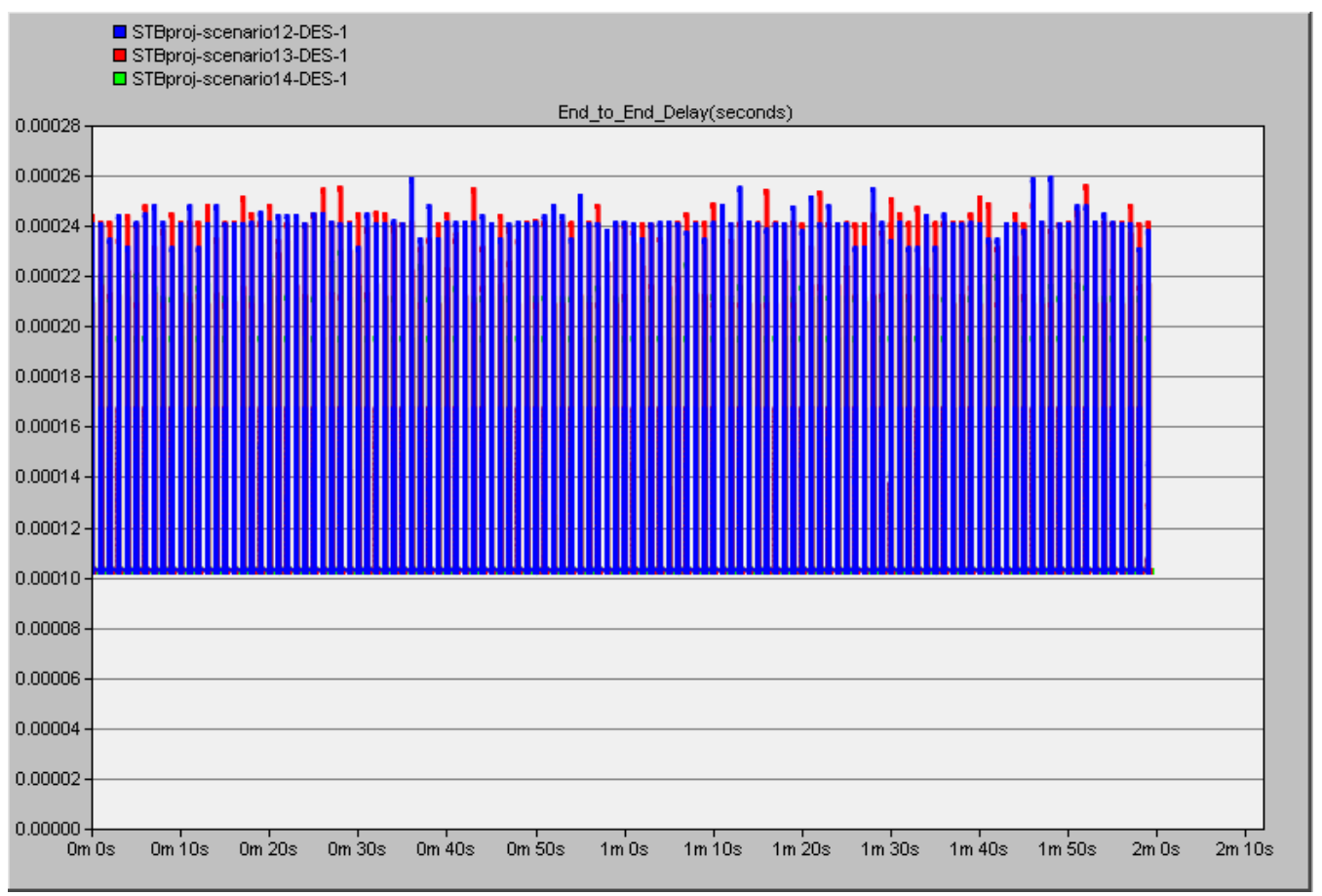

Figure 3. End-to-end delay of scenario 12 to scenario 14

To validate the performance of the broadcasting method in the network with various load, a series of scenarios are designed. In all the scenarios, network range is $1200 \mathrm{~m} \times 50 \mathrm{~m}$, distance of one hop 
is $600 \mathrm{~m}$, data packet length is $100 \mathrm{bit}$, date rate of transmitter and receivers is $10^{6} \mathrm{bps}$, interval time of node generate data packet is 1 packet per second. Nodes number of scenario 12, 13 and 14 are 40, 120, 240 respectively.

Experimental results are shown in figure 3 and table 3.

Table 3. Average number of received nodes

\begin{tabular}{|l|l|l|l|}
\hline No. of Scenario & 12 & 13 & 14 \\
\hline Average Number of Received Node & 29 & 88 & 178 \\
\hline
\end{tabular}

As shown in figure 3 and table 3, in network with various of network load, the end-to-end delay of the single-hop broadcasting method is stable and almost the same. The end-to-end delay is about $10^{-4}$ second, and most of the one hop range nodes could receive the broadcasted data.

\section{Conclusion}

In this paper, with OPNET network simulator, a single-hop broadcasting method based on busy tone is modeled and implemented. Experimental results show that in the various load network, performance of this broadcasting method is good.

\section{References}

[1] Ken Tang, Mario Gerla. MAC Layer Broadcast Support in 802.11 Wireless Networks. Proc. IEEE MILCOM 2000, 544-548(2000)

[2] Ken Tang, Mario Gerla. Random Access MAC for Efficient Broadcast Support in Ad Hoc Networks. Proc. IEEE WCNC 2000, 454-459(2000)

[3] Ken Tang, Mario Gerla. MAC Reliable Broadcast in Ad Hoc Networks. Proc. IEEE MILCOM 2001, 1008-1013(2001)

[4] Min-Te Sun, Lifei Huang, Anish Arora, Ten-Hwang Lai. Reliable MAC Layer Multicast in IEEE 802.11 Wireless Networks. Proc.ICPP'02, 527-536(2002)

[5] Wei Lou, Jie Wu. On reducing broadcast redundancy in ad hoc wireless networks. IEEE Trans. on Mobile Computing, 1(2):111-123(2002)

[6] Farzad Farnoud, Shahrokh Valaee. Reliable Broadcast of Safety Message in Vehicular Ad Hoc Networks. Proc. IEEE INFOCOM 2009(2009)

[7] Haas Z J, Deng J. Dual Busy Tone Multiple Access (DBTMA): A Medium Access Control for Multihop Networks. In: IEEE Transactions on Communications. Vol.50:975-985(2002) 